\title{
Copper Casting Variable Frequency Speed Control of Digital Control System
}

\author{
Chundi Jiang \\ college of electrical and information engineering, quzhou university, \\ Quzhou 324000, People's Republic of China \\ E-mail:cat6488@163.com
}

Keywords: digital control, horizontal continuous casting, traction motor

\begin{abstract}
This paper uses digital signal processor control horizontal continuous casting traction motor three states of positive rotation, inversion rotation and stop, to achieve the copper pull, stop, reverse thrust sport. At the same time, we can improve the yield, reduce the production cost by change the casting speed, the traction frequency and pitch.
\end{abstract}

\section{Introduction}

Today, China is the world's most important copper production, consumption and trade powers, but copper processing enterprise great majority is medium and small enterprises, equipment lags behind, takes the traditional continuous casting process, not only a waste of energy and can not be stepless speed regulation[1]. While the foreign popular adopts industrial control computer to control the AC servo motor drive type copper horizontal traction forming equipment, high cost, the maintenance requirements are high, it is difficult to promote in the small copper processing enterprises. So for the domestic enterprise's present situation, this thesis designs a high efficiency and low energy consumption, energy saving of copper horizontal continuous casting of variable frequency speed digital control system. Digital control with microprocessor as the core of the system, the complicated control circuit is realized by software, the hardware of the system is greatly simplified, and can make the speed static and dynamic performance of the system more perfect, system reliability, operability, and maintainability is greatly improved.

\section{System Process}

Continuous casting process refers to be cast with suitable temperature melt (copper) into the water cooling crystallizer, when the melt solidifies into a certain strength of the solidified shell, with the help of dummy bar and the traction rollers will have solidified billet continuously pulled out of the crystallizer, and when the material reaches its set length, will be synchronized automatically cut off [2, 3].

Traction motor rotational speed in each cycle the variation consists of four periods, as shown in Fig.1.

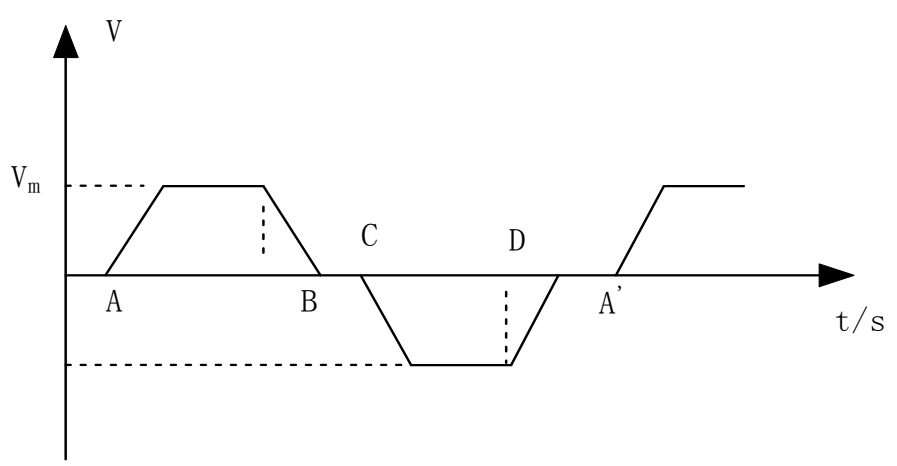

Fig. 1 traction speed change wave

In theFig.1, $V_{m}$ is the setting of the traction roller line speed, as shown in Eq.1 


$$
\mathrm{V}_{m}=\frac{2 \pi R(1-S) f_{m}}{p k_{n}}
$$

Type $\mathrm{R}$ for the traction roller radius, $\mathrm{P}$ motor pole pairs, $k_{n}$ is a mechanical reduction ratio, $\mathrm{S}$ for slip ratio of the motor, $f_{m}$ is the output frequency of the power supply for VVVF. Fig. 1 shows that the traction cycle $\mathrm{T}$ is:

$$
\mathrm{T}=t_{A B}+t_{B C}+t_{C D}+t_{D A}
$$

$t_{A B}$ is the time for traction, $t_{B C}$ is traction stop delay, $t_{C D}$ is time for thrust reverser, $t_{D A}$ is stop delay of the thrust reverser.

In Fig.1, the velocity waveform has two times the ascending and descending, which is composed of a VVVF power supply frequency lifting rate caused by. In order to adapt to the different shapes and different specifications of the needs of the production, to ensure product section metallographic quality and higher efficiency, for the period $\mathrm{T}$ choice should be able to do in a broad range of adjustable on line.

Traction pitch y is the billets are traction net long in a cycle,

$$
y=\frac{2 \pi R f_{m} t_{A B}(1-S)(1-\alpha)}{p k_{n}}
$$

$\alpha$ is the ratio of The time for thrust reverser and traction time, $t_{A B}$ and $f_{m}$ can be optimized, the remaining are structure constants.

\section{The Hardware Ddesign of Control System}

According to the traction mechanism control technology requirements and process parameters, this design uses the TI company for motor control and specially designed for TMS320LF2407A as digital control chip[4], The design power of a converter is $1000 \mathrm{~W}$, input from the keyboard, through the LF2407A display on LCD, Power driver circuit uses the driving module M579621L of the triangular company, Power tube using 1000V/15A IGBT---GT15J101, Using TBC20P current sensor to detect A phase and B phase current, Display module uses SMC1602ALCM LCD module, The final design of the copper molding machine traction motor digital control platform, function diagram shown as Fig. 2.

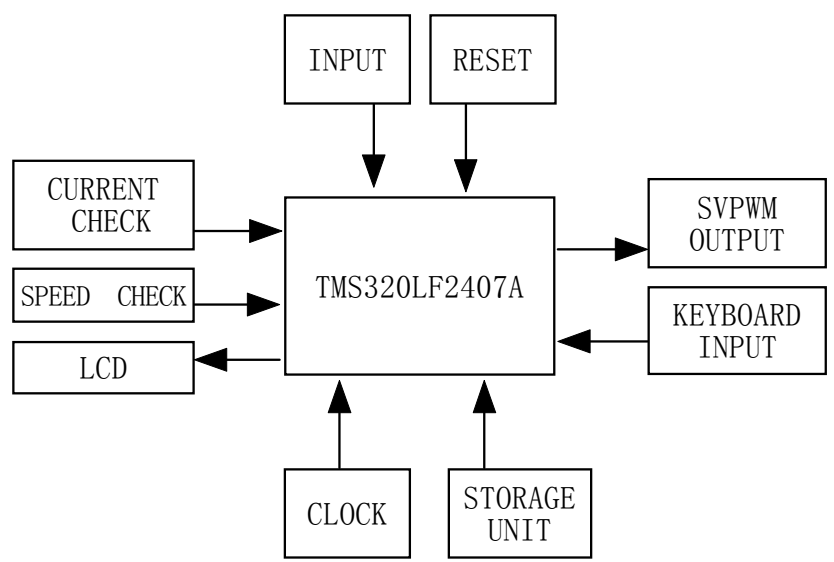

Fig. 2 the hardware function diagram of digital controller

\section{Software design of control system}

The DSP control software includes system initialization part and control part module function, the software structure diagram shown as Fig.3.The software consists of system initialization module, external interrupt processing module, timer interrupt processing module and serial interrupt service module, each module is composed of multiple sub modules [5]. Control system software part of the $\mathrm{C}$ language to write, some common subroutines using assembly language programming, and then curing the embedded into the main program, the main program is responsible for scheduling each 
sub module, the main program flow diagram shown as Fig. 4.

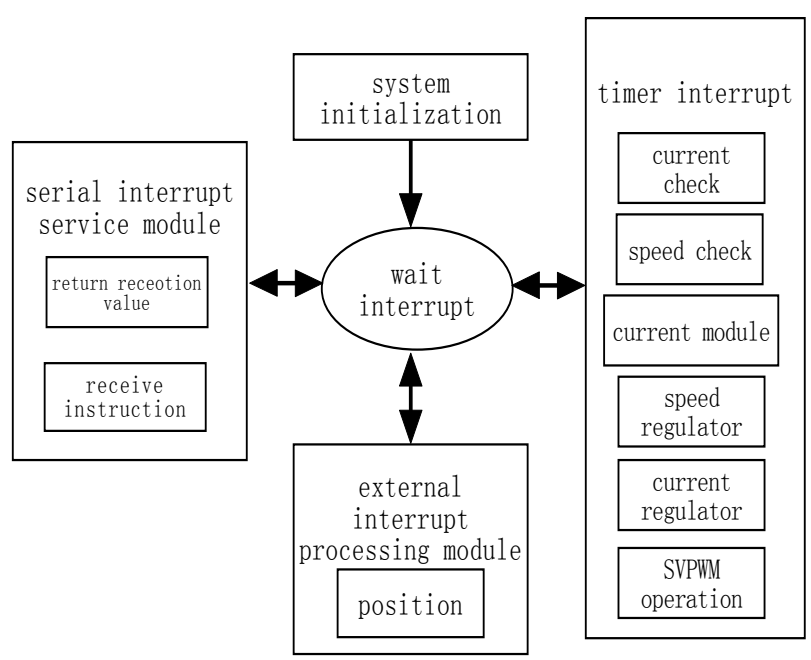

Fig. 3 DSP control system diagram

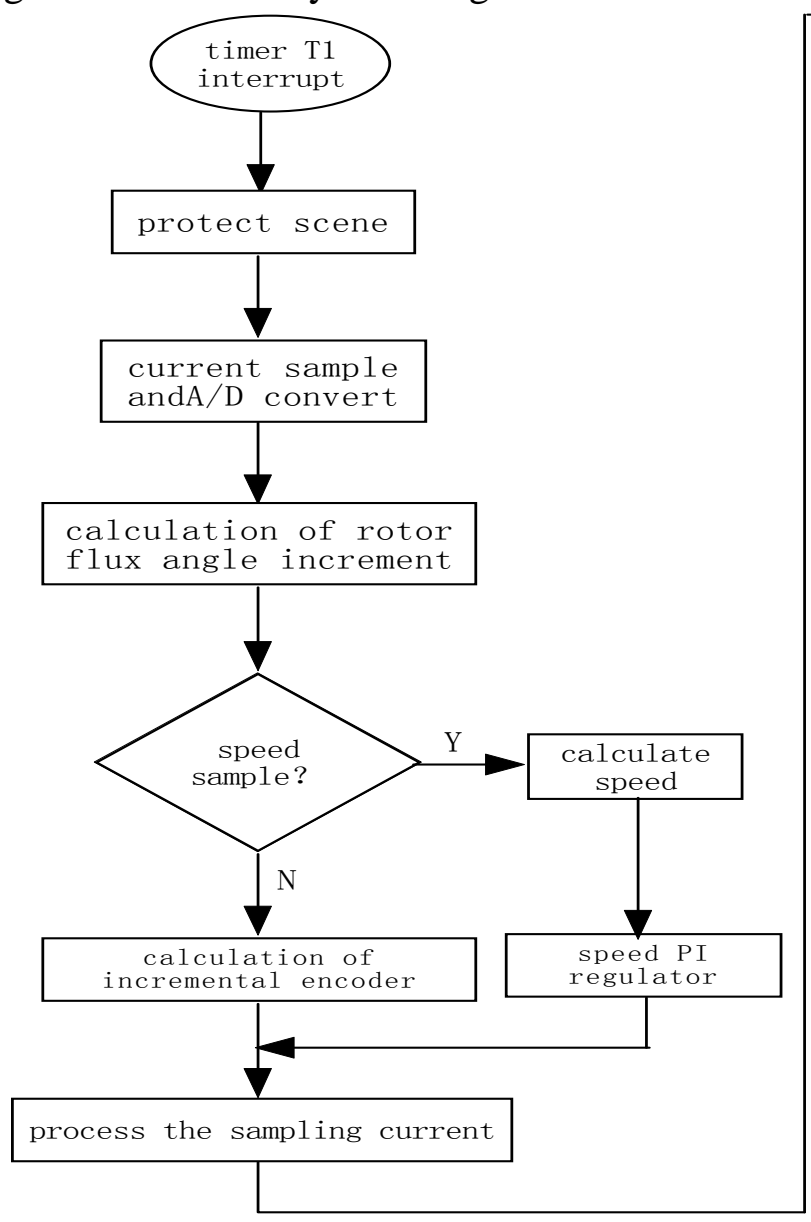

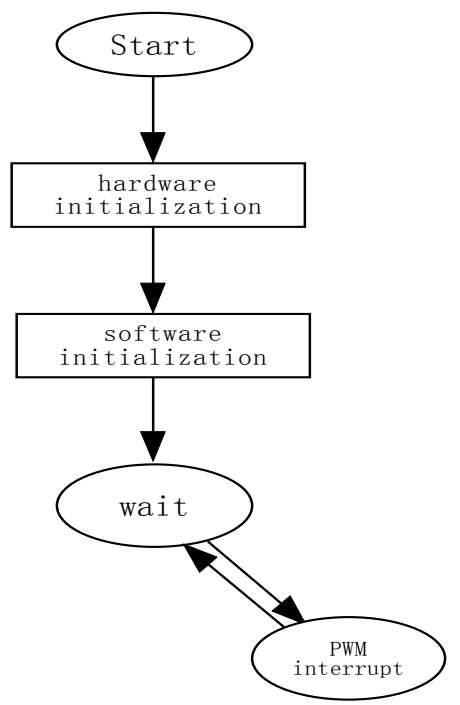

Fig.4 flow chart of main program

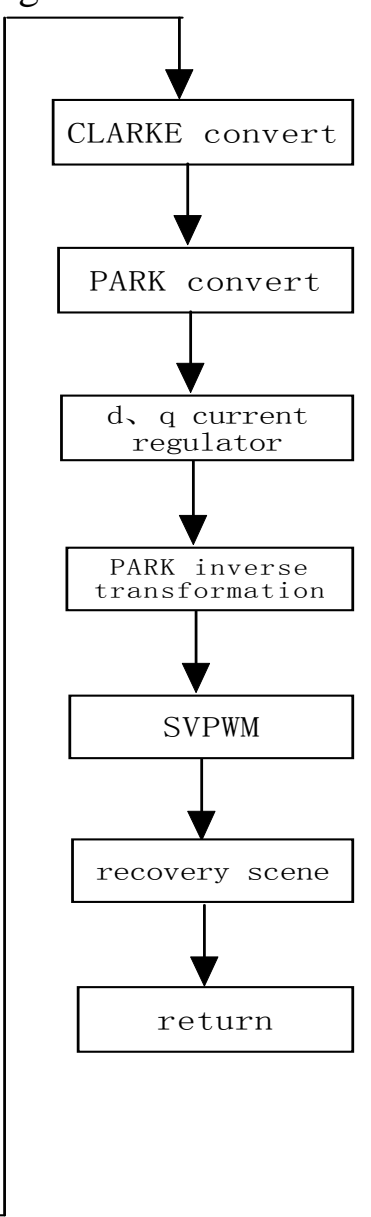

Fig.5 SVPWM underflow interrupt service subroutine diagram

Control system main program begins execution system initialization, the control register is initialized and related parameters setting, and then enter the cycle of waiting state. System initialization module completes the basic register settings and initialization of variables; External interrupt processing module according to the rotor position signal electronic phase change process; timer underflow interrupt processing module according to the position of the rotor, the stator current signal to adjust the speed, position regulator and current regulator; serial interrupt service module completes communication between the next-bit machine--- traction motor variable frequency speed control system and PC. When an interrupt is generated, programs response to the interrupts and execute the interrupt service subroutine. Interrupt service routines including SVPWM underflow 
interrupt service subroutine and external interruption protection subroutine, function of system control part mainly SVPWM underflow interrupt. Fig.5 shows the SVPWM underflow interrupt service subprogram flow chart.

\section{Conclusion and Prospect}

The design of digital signal processor controller realizes the copper traction control in four metre process, if using the digital signal processor controller SCI and SPI peripheral interface or CAN bus interface to realize the control system and the communication between host computer, can realize the network of production monitoring, and can also be integrated embedded operating system, which makes the system more expansion and portability, the man-machine interaction more convenient.

\section{References}

[1] Information on http://wenku.baidu.com/view/0c037633eefdc8d376ee3229.html

[2] L. Ding. Novel Copper Horizontal Continuous Casting and Traction Machine Design(China New Technologies and Products) vol 299-230(2011)

[3] W.Q. Zhang. The Technology Equipment Innovation and Control of Mechanical Failure of SCR Roller Caster (Special Casting and Nonferrous Alloys) Vol32 (5) 459-461(2012)

[4] J.Y. Liu, J.Q. Yang and Q.Z. Zhu: Application of PLC on Control System of Copper Cold Mill(Electrical Technology) vol 56-58(2009)

[5] H. Lu, C.R. Tong and Q.Y. Zeng. Research of Copper Smelter Automation System Based on Profibus(Control System) Vol 57-59(2009) 